\title{
BIOÉTICA, EDUCACIÓN FÍSICA Y DEPORTE: CRÍTICA A LAS EPISTEMES QUE ESCINDEN AL HOMBRE Y AL SABER
}

\author{
Fabio Zoboli* \\ Eduardo Francisco Freyre Roach ${ }^{* *}$ \\ Adolfo Ramos Lamar***
}

Resumen: Las Epistemes que escinden al hombre en cuerpo y alma, a los saberes, y los valores, promueven actividades de Educación Física y Deporte, que comprometen las expectativas Beneficiencia, No Maleficiencia, Autonomía y Justicia, que se sugieren en la Bioética. Revertir esta situación, favorecida por el status quo capitalista neoliberal globalizado, implica la asunción de enfoques epistemológicos de la corporalidad humana y su actividad motriz, en sus dimensiones sociales, culturales, etnico-raciales, de género, políticas, y ética. El trabajo aborda de forma crítica la concepción médico-biológica cartesiana de la Educación Física y del Deporte y las tensiones existentes entre bioética, educación física y actividad deportiva.

Palavras-Clave: Bioética. Educación Física. Deportes. Epistemes. Dualismo. Epistemología. Corporalidad.

Abstract: Sport has interests in "bioethics". The sport is losing ground to the enchantments of the scientific development and the capitalist economy of global market. The dual epistemes of physical education and a sport threat the bioethic expectations of beneficent, not malevolence, autonomy and justice. The phenomenologist-hermeneutic philosophical traditions, critical-dialectic, poststructuralists, and the complexity thoughts offer a epistemological and critical

* Doutor em Educação pela Universidade Federal da Bahia - UFBA. Professor do Departamento de Educação Física do Departamento de Educação Física da Universidade Federal de Sergipe - UFS.

** Professor da Universidade Agrária da Habana/Cuba. Pós-doutor em Educação pela Universidade Estadual de Campinas/SP - UNICAMP e Doutor em Filosofia pela Universidade de Moscou, Rússia.

** Pós-doutor em Educação pela Universidade de São Paulo - USP. Professor do Programa de Pós-graduação em Educação da Universidade Regional de Blumenau/SC - FURB. 
| 198 |

Bioética, educación física y deporte: crítica a las epistemes que...

perspective of dual epistemes and defend a applied bioethic to education and sciences of the sport. This scientific work discusses critically the medical-biological Cartesian view of Physical Education and Sports and the tensions between ethics, physical education and sports.

Keywords: Bioethic. Body Education. Sport.

\section{¿Bioética aplicada a la educación física y al deporte?}

El término de "Bioética" fue concebido por Renssenlaer Potter, aunque, según Engel (2004) se le atribuye también a Fritz Jahr. En sus escritos, Potter $(1971,1998,2005)$ se refiere a la bioética como un escenario de articulación entre las ciencias naturales, las sociales, la interdisciplinariedad, los valores (humildad, humanismo, y responsabilidad), y la interculturalidad, con el fin de potenciar los beneficios y revertir los perjuicios a la salud, al medio ambiente, y a la sobrevivencia, que emanan de los avances en las ciencias y tecnologías. Desde este punto de vista, la Bioética de Potter posee una connotación no sólo axiológica (relativa a los valores), sino también epistemológica (relativa a los saberes).

Los problemas bioéticos de la investigación biomédica con seres humanos y de la atención médica a la salud, durante gran tiempo, han estado acaparando gran atención. A esa "medicalización de Bioética", se le oponen hoy en día perspectivas teóricas que invitan a no perder de vista el sentido global que Potter le imprimió.

También están cobrando auge las perspectivas que se oponen a la monopolización del enfoque utilitarista, pragmático y neoliberal, que introdujo en la Bioética los principios de "Beneficiencia", "Autonomía", y "Justicia", pero interpretados a la luz de la relación contractual y mercantil (provedor-cliente) entre las personas, entendida como criterio moral supremo para la toma de decisiones en actividades que impactan los sobre la vida. Esta tradición filosófica postula que las personas, independientemente de sus diferencias, pueden tomar decisiones autónomas, auxiliándose por igual de principios morales abstractos.

La Educación Física y el Deporte son contextos donde se impone un análisis bioético, teniendo en consideración que, al 
igual que en la medicina, la investigación biológica y psicológica con seres humanos, la agricultura, y otros campos, se suscitan preocupaciones bioéticas. El trabajo aborda de forma crítica la concepción médico-biológica cartesiana de la Educación Física y del Deporte y las tensiones existentes entre bioética, educación física y actividad deportiva.

En este trabajo se fundamenta que la atención adecuada de esas preocupaciones implica la subversión de las epistemes, que escinden al ser humano en cuerpo y alma, a los conocimientos, y los valores, las ciencias y la ética.

\section{La educación física y el deporte: sus beneficios, pero también sus riesgos \\ El principio de la beneficencia alude a la expectativa ética} de promover el bien a la vida, mediante intervenciones que mejoren la salud y el bienestar. Este principio incluye también, el principio de no maleficencia (no dañar), inspirado en la Código de Ética de Hipócrates, y al que Beuchamp y Childress (2002) le atribuyen un status relativamente independiente. En el sentido utilitarista, pragmático, y neoliberal, el principio de beneficiencia tiene como condición fundamental para su aplicación casuística el cálculo de costos y beneficios de los actos.

Como muy bien se conoce, la teoría y la práctica de la educación física, el deporte, la cultura física terapéutica, y la recreación surgieron en función de la motivación por el cuidado del cuerpo y de los ejercicios físicos, su rendimiento, maximización u optimización. Se tratan de actos de intervención deliberada que influyen en la morfología de los diferentes sistemas del cuerpo humano: cardiovascular, respiratorio, renal, óseo, muscular, nervioso, endocrino, etc, donde como sabemos acontecen procesos físicos, químicos, mecánicos y energéticos.

En este sentido, no es difícil darse cuenta que la educación física y el deporte se acompañan no sólo de beneficios, sino también de riesgos para la salud, el bienestar y la calidad de la vida, asociados a la implementación de prácticas inadecuadas 
| 200 |

Bioética, educación física y deporte: crítica a las epistemes que...

de ejercitación física y de competición. Sin embargo, este análisis de la Educación Física y el Deporte desde el prisma de la expectativa de la beneficiencia y no maleficiencia, sería incompleto si se redujera solo a la dimensión de la "corporiedad motriz" del ser humano, o la salud, entendida en sentido biológico estrecho.

No se pierda de vista que la Educación Física y los Deportes, a través de la historia, responden también a determinados ideales de salud y educación, política, cultural y moral de los individuos. Por lo tanto, en el direccionamiento de estas actividades se inscriben intencionalidades que van más allá de las estructuras y dinámicas biológicas, como los incentivos asociados a la reputación, la autoestima, la imagen estética, y el desempeño de las personas, ya sea como miembro de una familia, ciudadano, estudiante, trabajador intelectual o manual, soldado, e inclusive como agitador político o religioso o amante sexual.

Las mismas se encuadran, a su vez, en un contexto socioeconómico, cultural y político determinado, encarnan en instituciones, y en prácticas. Por ejemplo, prácticas riesgosas en función del narcisismo estético corporal, se asocian a determinados ideales sobre la belleza humana introducidos por la ideología del consumismo y el hedonismo. Lo mismo pudiéramos decir, en el caso en que las políticas públicas y privadas priorizan los efectos económicos, políticos, simbólicos y militares del deporte y la educación física, y, por lo tanto, incentivan el afán de consumo, placer lúdico, y de records.

Merece también que se hable de cómo los sistemas de publicidad y organizaciones políticas y económicas del deporte participan en la reproducción de este enfoque, haciéndo énfasis en los triunfos del deporte (Betti, 2005), y ocultando las patologías anatómicas y fisiológicas que sufren los atletas. ¿Si realmente interesara la salud, por qué se ocultan los riesgos del deporte? ¿Llegará el día en que en la premiación de los equipos y de los países se tome en cuenta no sólo el triunfo deportivo, sino también la cantidad de lesiones que sufrieron, tanto los atletas que clasificaron, como los que se quedaron por el camino? ¿Qué responsabilidad ética asume un sistema deportivo con quienes 
por razones de salud o edad no pueden continuar practicando deporte y requieren de servicios que garanticen su "desentrenamiento"?.

No obstante, un análisis bioético, que esté atrapado en las redes del cartesianismo y el positivismo, y que, además sucumbe al principialismo utilitarista, que enfatiza en las relaciones de contrato mercantil entre las personas, al dar prevalencia al análisis costo y beneficios de las acciones, se inclina hacia los parámetros susceptibles de medición y calculo mediante números, estadísticas, y dinero.

\section{El respeto a la autonomía y el consentimiento informado de las personas}

El respeto a la autonomía se refiere a la participación activa de las personas en la toma de decisiones sobre las actividades que podrían beneficiar o perjudicar la calidad de su vida. Este principio presupone que se suministre a las personas información sobre su condición corporal, psíquica o ambiental, que se le solicite su consentimiento en los actos que recaen en esa condición, la protección de su privacidad, el anonimato, y su libertad de elección.

Este es un asunto de preferencia por autores que trabajan en el campo de la bioética aplicada a la educación física y el deporte. Por ejemplo, Botelho (2004), reporta que entre las 89 memorias de licenciatura en Educación Física (curso 1997-2002) de la Universidad del Estado de Río De Janeiro (UERJ), solo tres mencionaban el término de consentimiento, al menos solo una lo anexó; solamente se encontró una investigación con otros procedimientos éticos; y ninguna se sometió al Comité del Etica en la Investigación.

Similar resultado reportan Beck, De Almeida y Saldanha (2006) en su investigación realizada en la Universidad Federal de Santa Catarina. Detectan que la producción científica aumentó entre el 200 y el 2004 en un $78 \%$, pero con el sesgo de la ausencia de la aplicación de principios bioéticos. Los autores alegan que 
| 202 |

Bioética, educación física y deporte: crítica a las epistemes que...

2 artículos hacen mención de la garantía del anonimato, 5 se refieren a la solicitud del consentimiento informado, y muy pocos fueron sometidos a los Comités de Ética para la investigación científica.

Se viola la autonomía también cuando no se toman en cuenta la identidad psicológica, etnica, racial, de género, y clasista de las personas que realizan ejercicios físicos bajo una determinada orientación técnica. Considérese que las actividades de educación física no son neutrales a los estereotipos y estigmas sociales, y, por lo tanto, ya sea de forma explícita o sútil, pudieran tributar al perpetuamiento de discursos y prácticas discriminatorias. El trabajo de Brandao (2003) contiene un llamado de atención en este sentido.

Puede ocurrir también que se pasen por alto las preferencias, el derecho, las expectativas, las prioridades y la capacidad de tomar decisiones de los deportistas. Es el caso de cuando se viola su derecho humano a la autodeterminación, y no se le da participación activa en el entrenamiento, y peor aun cuando no se le dicen los riesgos del entrenamiento o de la competencia deportiva.

La educación física y el deporte corren este riesgo sobre todo cuando a las personas implicadas no se les suministra la suficiente educación, los datos, las informaciones y los conocimientos, que le podrían permitir una verdadera participación efectiva en la toma de decisiones que entrañan beneficios o perjuicios para su salud.

Sin embargo, esta cuestión debe ir mas allá de la violación del consentimiento informado, pues la autonomía no es un asunto tan simple que se resuelva en ese plano. Basta decir, que ese consentimiento puede ser manipulado para instrumentar un tipo de práctica deportiva, bajo determinados intereses, y aprovechando que los sujetos implicados y tomados como objetos o mercancias no están en condiciones de emitirlo adecuadamente.

Las sociedades y las culturas instituyen una determinada moral. Por eso, la expectativa bioética que atañe a la autonomía es una cuestión que se dirime por las representaciones morales 
que pautan la evaluación social del comportamiento de las personas como sujetos y seres sociales. La bioética se torna un asunto que entraña compromisos, acciones y opciones de competencia individual, comprendida a partir de su multiplicidad en la unidad de su condición de individuo, persona o sujeto autónomo.

A nuestro modo de ver, este principio bioético de la autonomía, aplicado a la educación física y a los deportes, debería implicar la responsabilidad no solo de todos los miembros de la sociedad con respecto al bienestar de las personas que realizan ejercicios físicos, sino también de ellas mismas, en cuanto a que las correcciones de las perturbaciones corporales autoinflingidas por negligencia, terminan siendo una carga para la familia y la sociedad.

Por eso Sérgio (2003 p. 31) tiene razón en decir lo siguiente:

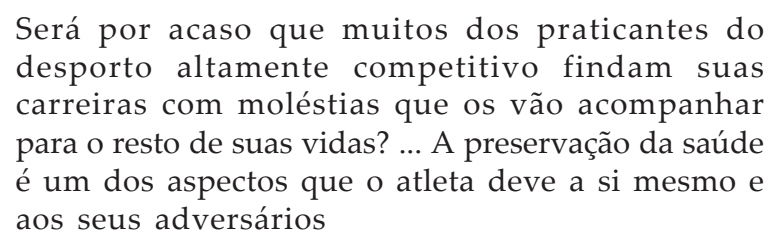

Esta reflexión relevante que nos hace este autor alude a que la cultura deportiva, como sistema colectivo de conocimiento y de conducta, debe estimular y fomentar en los actores sociales del deporte, el respeto por los otros y por sí mismo. Esta es una responsabilidad ética que se destina no sólo a entrenadores físicos y deportivos, sino también, como dijimos, a los deportistas y atletas, los científicos técnicos en educación física y deportes, a las familias y amistades, así como a los periodistas, los políticos del deporte, y al público de los espectáculos deportivos en general $\mathrm{y}$ en particular del deporte escolar.

Se tienen muchos testimonios archiconocidos sobre atletas que dando muestra de valentía y coraje, y que por ello son vistos como héroes, han sucumbido al dolor y la muerte. Podríamos 
| 204 |

Bioética, educación física y deporte: crítica a las epistemes que...

ilustrar esta situación trayendo a colación las siguientes interrogantes que nos ofrece Sérgio (2003, p. 12):

Não é o atleta de alta competição um trabalhador que vende ao clube a que pertence sua força de trabalho? Não é ele também humilhado e ofendido quando põe em risco a sua saúde, através de anestesias locais, que escondem, por poucas horas, lesões ósseas e musculares, de alguma gravidade?

Como es lógico, el éxito o el sacrificio suelen ocultar la "mala práctica" que ha cometido el atleta, en el ejercicio inadecuado de su autonomía, con anuencia de su entrenador, y peor aun del médico deportivo, y hasta del público que asiste al espectáculo deportivo.

Observese entonces que el principio de autonomía va más allá del asunto del privatismo moral, para abarcar dimensiones colectivas y sociales.

El acceso justo a los beneficios de la educación física y el deporte

Esta así llamada "trinidad bioética" se corona con el principio de la justicia que significa el acceso equitativo de todas las personas a los factores que se ofrecen para mejorar su vida. Según este principio es inaceptable todo tipo de discriminación, sea social, económica, cultural, racial, etaria o de género.

Cuando se discute la problemática de la justicia en el deporte, por lo general se trae a colación el fenómeno del "doping" o dopaje, es decir, la ingestión de drogas que al actuar sobre el metabolismo del atleta, a corto plazo hacen aumentar el rendimiento físico (Palacios, 1979), pero a mediado y largo plazo, desencadenan patologías agudas y crónicas. Con el doping se echa por tierra también el principio ético deportivo ancestral de la competencia leal y la simetría de posiciones y de oportunidades. Pero el análisis bioético en el deporte ha de ir más allá de estos límitesá.

Merece que se haga referencia a los casos en que los beneficios que reportan las actividades físicas y deportivas no se distribuyen de forma equitativa, es decir, que su acceso es injusto. Podríamos 
preguntarnos en que medida se promueve de forma desmedida fondos para el deporte "elite" de alto rendimiento, desatendiéndose la educación física o el deporte no profesional, amateur o masivo.

Vale la pena preguntarnos, si existe un orden equitativo de acceso a la educación física de calidad y de avanzada, a los deportes, a las instalaciones deportivas, a la recreación física, las actividades físicas de tiempo libre y placer, a la medicina deportiva, a los beneficios de las investigaciones e innovaciones en el campo de las ciencias de la educación física y de los deportes.

Interrogantes como estas se impone formularlas con relación a la política de educación física o deportiva nacional e internacional, e inclusive también cuando se trate de una determinada institución que presta servicios de este tipo o en el caso de las diferentes modalidades de deportes.

Esferas donde pueden darse injusticias son también la práctica de la selección de los atletas a razón de comportamientos favoritistas, elitistas o nepotistas. Lo mismo pudiéramos también decir en el caso de las competencias deportivas donde se cometen injusticias que benefician a unos y perjudican a otros, es decir, que se viola el principio ancestral de la rivalidad deportiva amistosa, pero también justa.

Entonces, la justicia no puede reducirse a la distribución equitativa de beneficios entre iguales, sino que implicaría traer a colación otros valores como la solidaridad, la caridad, la fraternidad, la amistad, el altruismo, la cooperación, y el sentido de humanidad.

La episteme de la escisión del hombre y del conocimiento: sus implicaciones bioéticas en la educación física y el deporte

Más allá también de las buenas o malas intenciones, así como de las improntas de la economía de mercado globalizada y del desarrollo científico-tecnológico, se nos presenta un tipo de "episteme" que reduce al hombre a su dimensión biológica, que lo escinde en cuerpo y alma, y que lo enajena del conocimiento. Se 
| 206 |

Bioética, educación física y deporte: crítica a las epistemes que...

trata de una episteme que, materializándose en el ámbito de la educación física y el deporte, potencia actos bioéticamente insostenibles.

En este contexto se visualizan otras escisiones referentes al saber: conocimientos-valores, ciencias naturales-ciencias sociales, explicación-comprensión, datos o análisis cualitativos-datos o análisis cuantitativos, conocimientos científicos-conocimientos no científicos, etc.

Este pensamiento dualista ha acompañado, por no decir que sigue acompañando, a las ciencias de la educación física y las ciencias del deporte, las cuales, en la búsqueda de sus bases conceptuales y epistemológicas, han participado de las ansias humanas de dominación de la naturaleza y la corporalidad. No hay más que tener en cuenta la tendencia exacerbada y aun vigente en nuestros días, de fundamentación médico-biológica de estas ciencias.

La episteme de la escisión mente-cuerpo, que ha tenido repercusión en la educación y el deporte ancla sus raíces históricas en el llamado dualismo cartesiano entre res cogitans e res extensa, cuya evolución entronca con la epistemología positivista proyectada hacia el aseguramiento y la legitimación de la educación física y el deporte en la Biología, la Cinesiología, la Anatomofisiología, y otras ciencias biológicas y médicas a fines. Esta concepción sustenta a una educación física y a un deporte biologizantes. Obrando de esta forma, la educación física y el deporte deshumanizan el sujeto, cuando en realidad lo humano es tal precisamente por poseer una dimensión compleja, tensionada constante y simbólicamente, a fin de (re)organizarse en su condición de sujeto histórico.

En términos de Sergio (2003), la educación física y el deporte, al fundarse en la episteme de la escisión mente-cuerpo, solamente visualizan el aumento de la velocidad, de la resistencia, de la fuerza y la potencia del cuerpo. Este autor filósofo lusitano agrega en su reflexión que esta perspectiva está muy lejos de la promoción de la salud, porque le está faltando un trabajo al nivel de la complejidad, que se estructure de acuerdo con un ego 
pensado y puesto al lado del ego multipensante, es decir, centrado más en la facticidad cuantitativa, menos en su realidad cualitativa.

Esta episteme es promovida también por el Positivismo, donde se postula la biologización de la educación física y el deporte, sobre la base de su coacción de la subjetividad en el hombre, dado obviamente a que esta se escapa de sus patrones o criterios de racionalidad científica. Es por eso que en las ciencias de la educación física y las ciencias del deporte se realizan una descomunal cantidad de estudios que reducen al hombre a aquello que es susceptible de observación, medición, experimentación y control, desconsiderándose con ello las dimensiones históricas y culturales de lo humano. Por consiguiente, un punto de vista reflexivo y crítico sobre esta dicotomía eclesiástica que escinden el ser humano en cuerpo y mente, consistiría en el rescate del cuerpo como aquella realidad que ha sucumbido a la esclavización o dosilización tecnológica.

Sin embargo la alternativa a esta episteme no consiste en una reivindicación del cuerpo en detrimento de la mente, sino de un nuevo entendimiento que dimensiones a uno u otro en los marcos de lo que se ha hecho llamar la corporalidad, como la instancia básica para pensar en las cuestiones bioéticas, que como ya se ha visto, suscitan la educación física y los deportes. Se trata de un giro radical respecto a las especulaciones intelectualistas sobre la determinación del ser del cuerpo. En este sentido, la educación física y el deporte en la actualidad están demandando una resignificación bioética por el respeto a la condición del cuerpo humano en la unidad de su ser que le es propia.

Entonces, el desafío tampoco consiste ente en unir lo que fue separado, sino más bien en pasar a cuestionar aquellas determinantes intelectuales que han incidido en el olvido de la unidad humana y de si corporalidad.

Merece la pena pensar en lo que Alves in Bruhns (1986, p. 41), cuando al referirse a la educación física y sus modos de tratamiento del cuerpo, escribe: 
| 208 |

Bioética, educación física y deporte: crítica a las epistemes que...

\begin{abstract}
Quanto a mim, valorizo o sono tranqüilo, coisa que nunca se celebrou nas Olimpíadas, mas que deveria ser um dos grandes direitos universais dos seres humanos. Poucas pessoas, neste mundo, irão jamais correr os 100 metros rasos, porém todos têm que dormir... Mas não me consta que coisas relativas ao dormir bem se encontrem nos currículos da Educação Física. Talvez porque não se considere que o corpo dormindo seja corpo.
\end{abstract}

Las epistemes que escinden el hombre en cuerpo y mente, y también a los conocimientos en naturales y sociales, en científicos y no científicos, se articulan con aquella otra episteme aun vigente que sugiere el entendimiento del cuerpo como una maquina, la "taylorización" o la producción en serie de cuerpos en el campo de la educación física y los deportes. En términos de Partisans (1988, p. 27): “El robot del deporte está a punto de nacer". Esto significa que se elige la técnica que resulta más eficaz y rentable, pero a costa de tratar al cuerpo sólo desde un ángulo instrumental, reduciéndolo a la categoría de artefacto mecánico que obedece a las leyes de la cinemática y de la gravedad. La maximización de las potencialidades del cuerpo sobrepasa su optimización ilimitada, lo cual hace evidente la presencia de una voluntad o ímpetu de poder sobre los cuerpos. Con ello también en la educación física y en el deporte los engranajes del "hombre máquina" permanecen bien reguladas a los engranajes de la ciencia, la tecnología y el poder, articuladas a las instituciones y las costumbres, y que hacen girar la industria, el mercado y el dinero, tributando así a la lógica del capital.

El método mecanicista y racionalista sugerido por la episteme positivista ofrece la "columna vertebral" no solo para la educación física y los deportes, sino también para otras áreas como la medicina. De ahí que en todas estas áreas emerge un conjunto de procedimientos ascéticos para el control de las mentes y los cuerpos, articulados a una estructura tecnológica. Ese conjunto disciplinar y necesario esta ligado a toda una estructura tecnológica y científica que se encuentra imbricada a la lógica de la economía de mercado globalizada y en función del capital.

Podemos afirmar que de cierto modo, el deporte actual visualizado desde la episteme del hombre-máquina - está repleto de 
ciborgues ${ }^{1}$, es decir, que produce máquinas atléticas, fabricadas y subjetivadas, magníficos seres orgánicos criados en laboratorios que pueden ser también considerados criaturas o engendros de la ciencia y del capitalismo. Esto no es más que una fusión tensada, intercedida por una lucha de significados que transitan entre el poder, el placer y la tecnología. En este sentido citemos a Kunzru (in Silva, 2000, p. 26):

\begin{abstract}
Vencer os Jogos Olímpicos na era do ciborgue não tem a ver simplesmente com correr mais rápido. Tem a ver com a interação entre medicina, dieta, práticas de treinamento, vestimentas e fabricação de equipamentos, visualização e controle de tempo. Tem a ver com a ciborguização de atletas por meio de drogas para melhorar a performance. Com drogas ou sem drogas, o treinamento e a tecnologia fazem do atleta olímpico um nó em uma rede tecnocultural internacional tão "artificial" quanto o super corredor Ben Johnson no ponto máximo de consumo de esteróides.
\end{abstract}

Es decir, de un lado el organismo - carne y subjetividad que de vez en vez se artificializan, por otro lado la maquina, como una amontonamiento de engranajes, simuladores de características humanas.

\title{
Las epistemes alternativas y su entronque con la bioética
}

Estudios realizados por Chávez (2005), Silva (2006), y Gamboa (2007), constatan como hoy en día predominan filosofías que arremeten contra las epistemes dualistas, que como vimos coartan el camino para una bioética de la educación física y el deporte, y también para las pretensiones de identidad epistemológica de la propia bioética. Es el caso de las epistemología de la educación física que se sustenta en los

${ }^{1}$ El término de ciborgue deriva de cyborg, abreviatura de "cybernetic organism". Kybernetes, que em griego antiguo alude al "hombre que dirige". 
| 210 |

Bioética, educación física y deporte: crítica a las epistemes que...

abordajes fenomenológico-hermenéutico, crítico-dialéctico, sestructuralistas, y los inspirados en la teoría de la complejidad. También se cuenta con investigaciones sobre las cuestiones de ética que suscitan la educación y el deporte (Benghi y Zoboli, 2004 y Silva y Zoboli, 2006), cuando caen bajo la trampa de las epistemes dualistas.

Conceptos propuestos por la tradición filosófica fenomenológico-hermenéutica como "intencionalidad de la conciencia" y "mundo de la vida" (Husserl), "ser-en-el-mundo" (Heidegger), y "corporalidad" (Merleau-Ponty), encierran toda subversión de las epistemes dualistas, biologistas y mecanicistas sobre el hombre, su cuerpo, el conocimiento y las ciencias, así como de la relación sujeto-objeto, el hombre y el mundo. Esta tradición sugiere que en la configuración de las ciencias de la educación física y los deportes, se exploren las vivencias, las experiencias, las emociones, las significaciones subjetivas o sentidos subjetivos individuales, y valores, compartidos intersubjetivamente por las personas que hacen ejercicios y hacen deporte. Se busca entonces una objetividad no pautada absolutamente por las magnitudes cuantitativas en que se expresan los fenómenos humanos, sino que toma en consideración la implicación de las subjetividades. La corporalidad humana se enfoca como totalidad u unidad mente-cuerpo, la mente no queda reducida a su dimensión racional y el cuerpo no queda reducido a sus dimensiones biológicas y mecánicas (Freyre, 2007).

Por su parte, la filosofía o Teoría Crítica del Jürguen Habermas, denuncia la racionalidad instrumental y burocrática que se esconde tras el cientificismo cartesiano y positivista. Como alternativa se sugiere una educación física pautada no por un interés empírico-analítico, sino más bien crítico-dialéctico, centrado no sólo en la intersubjetividad "dialógica"y argumentativa de los sentidos (incluyendo el axiológico y ético) que los prácticantes del deporte atribuyen y negocian en su praxis física o deportiva, sino también, al desmantelamiento de las relaciones de dominación y de poder que potencian, que al fin y 
al cabo, ponen en riesgo las expectativas bioéticas. Esta filosofía crítica, que articula puntos de vistas de Marx y de Freud, exige el entendimiento de una corporalidad enredada en la praxis social de los sujetos, donde tanto la mente como el cuerpo quedan a merced de la totalidad social. Desde este punto la educación física y el deporte escaparían de la estrechez en que las epistemes dualistas amenazan con encerrarlas (Chávez, 2005).

Esta conexión que existe entre las epistemes dualistas, biologistas, cientificistas y mecanicistas, y los juegos de poder que objetivan y subjetivizan a los individuos, es también criticada por el Post-estructuralismo. El gesto desconstruccionista que propone Jacques Derridá no es más que una reacción contra un pensamiento que el llama dialectizante y que consiste en el establecimiento de relaciones de binariedad, contraste, oposición y jerarquía entre los términos, por ejemplo, entre cuerpo y alma. En cuanto a Michel Foucault merece destacarse que la conceptualización de las epistemes dualistas, ha de entenderse en asociación al afán de docilización del cuerpo, inscrita en los sus juegos de saber y poder, en función del mecanismo de reproducción económica del capital (Zoboli, 2003).

La epistemología de la educación que se desprende de la Teoría de la Complejidad (Polanco, 2006) propuesta por autores como Edgar Morin, apunta hacia una concepción integradora de la corporalidad humana que la libera de las trampas reducionistas, dualistas, anatatomizantes o atomizantes, parcelarias, y simplificantes del cartesianismo y el positivismo. $\mathrm{Al}$ respecto en su Los siete saberes necesarios para la educación del futuro) plantea:

El ser humano es a la vez físico, biológico, síquico, cultural, social, histórico. Es esta unidad compleja de la naturaleza humana la que está completamente desintegrada en la educación a través de las disciplinas y que imposibilita aprender lo que significa ser humano. Hay que restaurarla de tal manera que cada uno desde donde esté tome conocimiento y conciencia al mismo tiempo de su identidad compleja 
| 212 |

Bioética, educación física y deporte: crítica a las epistemes que...

y de su identidad común a todos los demás humanos (Morin, 1999:19).

La perspectiva de la Educación Corporal confluyen con las anteriores perspectivas epistemológicas, en la medida que:

... excede el cerco disciplinar de la Educación Física y
puede promoverse, por ejemplo, para la educación
del autogobierno de las emociones violentas, la
internalización de una sexualidad planificada y
responsable, el cuidado del propio cuerpo o la
promoción de regímenes alimenticios preventivos
de la salud... Nos invita a revi-sar los contenidos
tradicionales de la Educación Física - deporte, juego,
gimnasia, natación y vida en la naturaleza-y exceder
los tratamien-tos instrumentales de meras
actividades físicas evaluación de su relevancia y
significatividad social (Cachorro, 2008: 74-76).

Esto significa tomar en cuenta los elemen-tos estructurales y las aristas subjetivas, sociales, políticas, y culturales, que se inscriben en la corporalidad.

Y lo mismo vale para la Bioética, que en la connotación global que le imprimiera Potter - versus versión medicalista inspirada en la tardición utilitarista y pragmática - implica integrar el mundo del hombre, su saber, desear y conocer, abordar ese mundo a partir de un prisma interdisciplinar, que articule conocimientos y valores, en una dimensión participativa, es decir, que involucra a toda la sociedad (Delgado, 2002). En este sentido, de la episteme bioética se infiere un posicionamiento crítico respecto a los sesgos que acompañan a las epistemes que escinden al hombre, a los saberes, y valores.

\section{Concluyendo}

Si bien los principios de Beneficiencia, Autonomía, y Justicia expresan verdaderas preocupaciones éticas que suscitan los actos 
de intervención en la vida, bajo el prisma de las epistemes dualistas, y también del principialismo utilitarista y neoliberal, el énfasis recae en una relación instrumental, contractual, mercantil, y monetaria, desde donde se valoran las expectativas bioéticas.

Las epistemes alternativas, en la medida que ponen en entredicho a las epistemes dualistas, sugieren otro escenario de teorización de las cuestiones bioéticas que suscita la Educación Física y el Deporte, donde se toman las expectativas bioéticas (no reducidos a los que postula el principialismo utilitarista, pragmático y neoliberal) a la luz de un entendimiento de la corporealidad en relación con el contexto de significados subjetivos e intersubjetivos, la totalidad social compleja, y las relaciones de dominio y poder.

Asumir las epistemes alternativas (incluyendo la que emana de la misma Bioética) en el enfoque bioético de la Educación Física y el Deporte implica comprender y tratar el cuerpo de las personas, pero en función de las mismas, y entendidas como sujetos, no como objetos susceptible de manipulación instrumental. Entonces sería mejor hablar no del cuerpo per se, sino de las personas que tienen cuerpo, es decir, de corporalidad.

Por eso, con independencia de las controversias que existen sobre las diferencias entre Educación Física y Deporte, ciencias del deporte y ciencias de la educación física consideramos que el deporte tiene que ver con la incidencia en las potencialidades corporales de las personas, consideradas como deportistas o atletas, pero en todas las aristas que la conforman, y en relación a su historicidad y cotidianiedad. Lo mismo para las personas que se involucran en otras actividades físicas.

Colocar el análisis bioético de la Educación Física y el Deporte en la perspectiva de estas epistemes alternativas implica subvertir la reducción de la beneficiencia a las estrechas nociones de costos y beneficios en sentido objetivista, biológicoreduccionista, cuantitativista, estadístico, y monetario.

Significa también entender la autonomía no en sentido privatista u egocéntrico, sino en una perspectiva intersubjetiva, 
| 214 |

Bioética, educación física y deporte: crítica a las epistemes que...

dialógica, intercultural, y política. A fin de cuentas, se saber perfectamente que es posible manipular el criterios de las personas, ya sea ocultándole información, o suministrarle demasiada, o no acorde a su capacidad de apreciación y ponderación de las circunstancias.

Y, por último, se exigiría un enfoque donde la discusión sobre la justicia conlleve al reeplanteamiento crítico de las relaciones que inscribe la corporalidad humana en relaciones asimétricas y dispositivos de poder o dominio. La justicia despojada del enfoque utilitarista, neoliberal y contractualista (el igual que el enfoque paternalista que se deriva de la episteme dualista cartesiana y positivista), involucra otras dimensiones de la justicia y moralidad en su conjunto, como por ejemplo, la globalización de la solidaridad y la ayuda altruista que hoy se requiere para erradicar las desigualdades sociales en materia de salud pública, alimentación, seguridad social, y de educación física y deporte.

\section{Referência}

ACOSTA S. J. R. Bioética para la sustentabilidad. Publicaciones Acuario, Centro Félix Varela, La Habana, 2002.

ALVES, R. O corpo e as palavras. In: BRUHNS, Heloísa Turini. (org.) Conversando sobre o corpo. p. 17-42. Campinas, SP: Papirus, 1986.

BECK D. C. C, de Almeida Gomes Marcius, y Saldanha Diniz Maria. Bioética em pesquisa na Educação Física. Revista Digital Buenos Aires - Año 11 - No 96 - Mayo de 2006. In: http://www.efdeportes.com/efd96/bioetica.htm Acesso em 11 septembro de 2010.

BENGHI R. y ZOBOLI F. Educação Física e promoção humana. Acadêmica, Blumenau-SC, 2004.

BETTI, M. A janela de vidro: esporte, televisão e educação física. Papirus: Campinas, 2005. 
BEAUCHAMP, Tom L.; CHILDRESS, James F. Princípios de ética biomédica. São Paulo: Loyola, 2002.

BRANDÃO, Claudio. Bioética na educação física. Maceió: Edições Catavento, 2003. BRANDÃO, Claudio. Bioética na educação física: discriminação em uma escola pública do Distrito Federal. 2002. 94 f. Dissertação (Mestrado em Educação).

BOTELHO, Rafael Guimarães. Bioética no contexto das pesquisas científicas brasileiras em educação física e ciências do desporto. Revista Portuguesa de Ciências do Desporto, v. 4, n. 2, p. 127-128, set. 2004. Suplemento.

CACHORRO Gabriel. Ideas para pensar la Educación Física. Revista Educación física y deporte, n. 27-2, 65-75, 2008, Funámbulos Editores.

CHAVES M. A produção do conhecimento em educação física nos estados do nordeste(Alagoas, Bahia, Pernambuco e Sergipe, 1982-2004. Salvador, 2005. Relatorio de Posdoutorado, Universidade Federal da Bahia.

DÍAS J. M. Bioética e Educação Física. Documento CONFEF/ CREF. www.confef.org Acessado em 30 out/2005.

ENGEL, E-M. O desafio das biotécnicas para a ética e a Antropologia. Veritas 2004; 50(2):205-228. http://www.ufrgs.br/bioetical bioet27.htm. Acesso em 12 de janeiro de 2010.

FREYRE R. E. F. A abordagem fenomenológico-hermenêutica na produção de teses de doutorado em educação no estado de São Paulo. Relatório de Pós-doutorado, UNICAMP, 2007.

GAMBOA S. S. A. Epistemologia da Educação Física: as interrelações necessárias. 2007 (Inédito).

KUNZRU, H. "Você é um ciborgue": Um encontro com Donna Haraway. In SILVA, Tomaz Tadeu. Antropologia do ciborgue: as vertigens do pós-humano. pp. 19-37. Belo Horizonte: Autêntica, 2000. 
MORIN EDGAR. Los sietes saberes necesarios para la educación del futuro. Organización de las Naciones Unidas para la Educación, la Ciencia y la Cultura. París, 1999.

PALACIOS, S. M. Doping y dopaje: una guía para médicos y entrenadores. Buenos Aires, 1979.

PARTISANS, J. Deporte, cultura y represión. Barcelona, Editorial Gustavo Gil, 1988.

POLANCO B., Yanet. La Epistemología de la complejidad como recurso para la educacion. Revista Ciencias de la Educación Crítica. Año 6,?Vol. 1, ? N. 27, Valencia, Enero-Junio 2006, p. $179-188$.

POTTER R. Bioética Puente, Bioética Global y Bioética Profunda. 1998 In: http://165.158.1.110/spanish/hdp/PRB/pot.htm. Acesso em 14 de junho de 2010.

. Deep and global Bioética for a livable third millennium. In: The Scientist (12/1/:9, Jan. 2005). 1971.

. Bioethics, bridge to the future. Prentice Hall: New York,

SÉRGIO, M. Algumas teses sobre o desporto. 2 ed. Lisboa - Portugal: compendium, 2003.

SILVA A. N. M. y F. Z. Educação e Ética: Historicidade, práxis e processos formativos. Blumenau/SC: Edifurb, 2006.

SILVA de S. R. V. Pesquisa em educação física: determinações históricas e implicações epistemológicas. Tese (doutorado) 2006. UNICAMP.

ZAMORA RAMÍREZ MONICA. Aproximación a una concepción bioética del deporte. In: http://www.cbioetica.org/revista/53/ 531417.pdf. Acesso em 11 septembro de 2010.

ZOBOLI ELCP. Bioética e Atenção Básica: um estudo de ética descritiva com enfermeiros e médicos do Programa Saúde da Família. Tese de 
Doutorado, Faculdade de Saúde Pública da Universidade de São Paulo. São Paulo 2003.

Idéias $\mid$ Campinas(SP) | n. 2 | nova série $\mid 1^{\circ}$ semestre (2011) 